\title{
A robust structured preconditioner for time-harmonic parabolic optimal control problems
}

\author{
Zhao-Zheng Liang ${ }^{1,3}$ • Owe Axelsson ${ }^{2,3}$. \\ Maya Neytcheva ${ }^{3}$
}

Received: 9 August 2017 / Accepted: 22 November 2017 / Published online: 5 December 2017 (C) The Author(s) 2017. This article is an open access publication

\begin{abstract}
We consider the iterative solution of optimal control problems constrained by the time-harmonic parabolic equations. Due to the time-harmonic property of the control equations, a suitable discretization of the corresponding optimality systems leads to a large complex linear system with special two-by-two block matrix of saddle point form. For this algebraic system, an efficient preconditioner is constructed, which results in a fast Krylov subspace solver, that is robust with respect to the mesh size, frequency, and regularization parameters. Furthermore, the implementation is straightforward and the computational complexity is of optimal order, linear in the number of degrees of freedom. We show that the eigenvalue distribution of the corresponding preconditioned matrix leads to a condition number bounded above by 2 . Numerical experiments confirming the theoretical derivations are presented, including comparisons with some other existing preconditioners.
\end{abstract}

Keywords PDE-constrained optimization - Time-harmonic parabolic equation • Preconditioning $\cdot$ Iterative solution method $\cdot$ Spectral analysis

Maya Neytcheva

maya.neytcheva@it.uu.se

Zhao-Zheng Liang

liangzhzh2014@1zu.edu.cn

Owe Axelsson

owe.axelsson@it.uu.se

1 School of Mathematics and Statistics, Lanzhou University, Lanzhou, People's Republic of China

2 Institute of Geonics, Czech Academy of Sciences, Ostrava, Czech Republic

3 Department of Information Technology, Uppsala University, Uppsala, Sweden 


\section{Introduction}

Consider the following optimization problem: find the state $y(x, t)$ and the control $u(x, t)$ that minimize the cost functional

$$
J(y, u)=\frac{1}{2} \int_{0}^{T} \int_{\Omega}\left|y(x, t)-y_{d}(x, t)\right|^{2} d x d t+\frac{\beta}{2} \int_{0}^{T} \int_{\Omega}|u(x, t)|^{2} d x d t,
$$

subject to the time-periodic parabolic problem

$$
\begin{aligned}
\frac{\partial}{\partial t} y(x, t)-\Delta y(x, t) & =u(x, t) \text { in } Q_{T}, \\
y(x, t) & =f(x, t) \text { on } \Sigma_{T}, \\
y(x, 0) & =y(x, T) \text { in } \Omega, \\
u(x, 0) & =u(x, T) \text { in } \Omega .
\end{aligned}
$$

Here $\Omega$ is an open and bounded domain in $\mathbb{R}^{d}$ for $d \in\{1,2,3\}$ with Lipschitzcontinuous boundary $\Gamma, Q_{T}=\Omega \times(0, T)$ is the space-time cylinder and $\Sigma_{T}=\Gamma \times$ $(0, T)$ is its lateral surface. Further, $y_{d}(x, t)$ is a given target state function and $f(x, t)$ is a given function related to Dirichlet boundary condition, $\beta>0$ is a regularization parameter. We assume that $y_{d}(x, t)$ and $f(x, t)$ are both continuous functions in $Q_{T}$ and are $T$-periodic in time.

Problems of the form (1.1)-(1.2) belong to the field of PDE-constrained optimization and appear when modeling and simulating numerous complex natural phenomena. For details of the theoretical aspects and the application of numerical optimization techniques for the solution of PDE-constrained optimization problems, see, for instance, $[14,18,31]$. In this paper, our primary interest is the fast and robust solution of the large-scale matrix systems arising after discretizing the problem (1.1)-(1.2).

Similarly to $[5,21]$, we assume further that $y_{d}(x, t)$ and $f(x, t)$ are time-harmonic, i.e.,

$$
y_{d}(x, t)=y_{d}(x) e^{i \omega t} \text { and } \quad f(x, t)=f(x) e^{i \omega t}
$$

with $\omega=\frac{2 \pi k}{T}$ for some positive integer $k \in \mathbb{Z}$. Then, there is a time-periodic solution to the original control problem of the form

$$
y(x, t)=y(x) e^{i \omega t} \quad \text { and } \quad u(x, t)=u(x) e^{i \omega t},
$$

where $y(x)$ and $u(x)$ are the solutions of the following optimal control problem:

$$
\min _{y, u} \frac{1}{2} \int_{\Omega}\left|y(x)-y_{d}(x)\right|^{2} d x+\frac{\beta}{2} \int_{\Omega}|u(x)|^{2} d x,
$$

subject to

$$
\begin{aligned}
i \omega y(x)-\Delta y(x) & =u(x) \text { in } \Omega, \\
y(x) & =f(x) \text { on } \Gamma .
\end{aligned}
$$

For the numerical solution of the problem (1.5)-(1.6), we use the discretize-thenoptimize approach, which is plausible as the problem is coercive. For details on this, we refer, for instance, to [12]. After discretizing the problem using equal order finite element spaces, we obtain the following finite dimensional problem:

$$
\min _{y, u} \frac{1}{2}\left(y-y_{d}\right)^{*} M\left(y-y_{d}\right)+\frac{\beta}{2} u^{*} M u
$$


subject to

$$
i \omega M y+K y-M u=f .
$$

Here $M$ and $K$ are the mass and stiffness matrices, representing the $L^{2}$-inner product and the discretized negative Laplacian, respectively. The vectors $y, y_{d}, u$, and $f$ denote, respectively, the discretized versions of $y, y_{d}, u$, and $f$, i.e., coefficient vectors of the corresponding expansions in terms of the finite element basis functions used.

A simple and general approach to solve the so-obtained constrained optimization problem is to construct the Lagrangian functional

$$
\mathscr{L}(y, u, p)=\frac{1}{2}\left(y-y_{d}\right)^{*} M\left(y-y_{d}\right)+\frac{\beta}{2} u^{*} M u+p^{*}(i \omega M y+K y-M u-f),
$$

where $p$ denotes the Lagrange multiplier, associated with the constraints, and apply the first-order necessary optimality conditions, or Karush-Kuhn-Tucker (KKT) conditions, for stationarity. In detail, the first-order conditions are $\nabla \mathscr{L}(y, u, p)=0$, leading to the following block system of linear equations:

$$
\left[\begin{array}{ccc}
M & 0 & K-i \omega M \\
0 & \beta M & -M \\
K+i \omega M & -M & 0
\end{array}\right]\left[\begin{array}{l}
y \\
u \\
p
\end{array}\right]=\left[\begin{array}{c}
M y_{d} \\
0 \\
f
\end{array}\right]
$$

The second row in (1.7) implies that $u=\frac{1}{\beta} p$. In this particular problem formulation we can, hence, eliminate the Lagrange multiplier $p$ and reduce (1.7) to

$$
\left[\begin{array}{cc}
M & \beta(K-i \omega M) \\
K+i \omega M & -M
\end{array}\right]\left[\begin{array}{l}
y \\
u
\end{array}\right]=\left[\begin{array}{c}
M y_{d} \\
f
\end{array}\right] .
$$

A simple scaling of the latter system leads to the following optimality system:

$$
\mathscr{A}_{\mathrm{I}}\left[\begin{array}{l}
y \\
\tilde{u}
\end{array}\right]=\left[\begin{array}{c}
M y_{d} \\
\widetilde{f}
\end{array}\right] \text { with } \quad \mathscr{A}_{\mathrm{I}}=\left[\begin{array}{cc}
M & \sqrt{\beta}(K-i \omega M) \\
\sqrt{\beta}(K+i \omega M) & -M
\end{array}\right],
$$

$\widetilde{u}=\sqrt{\beta} u$ and $\widetilde{f}=\sqrt{\beta} f$. In addition, the linear system (1.8) can also be equivalently transformed to

$$
\mathscr{A}_{\mathrm{II}}\left[\begin{array}{l}
y \\
\widehat{u}
\end{array}\right]=\left[\begin{array}{c}
M y_{d} \\
\widetilde{f}
\end{array}\right] \text { with } \quad \mathscr{A}_{\mathrm{II}}=\left[\begin{array}{cc}
M & -\sqrt{\beta}(K-i \omega M) \\
\sqrt{\beta}(K+i \omega M) & M
\end{array}\right]
$$

and $\widehat{u}=-\sqrt{\beta} u$. Compared with $\mathscr{A}_{\mathrm{I}}$, the coefficient matrix $\mathscr{A}_{\mathrm{II}}$ loses the Hermitian property, but gains positive definiteness. This can be advantageous when using certain Krylov subspace methods, such as restarted GMRES; see [29, 30]. Linear systems of the form (1.8) and (1.9) belong to the class of saddle point problems. For a detailed account of the theory related to solution methods and preconditioning techniques for saddle point problems, we refer to $[1,8,11,24,25]$, to mention a few, and to [32] for more general block-factorization preconditioners. Owing to the large dimensions and sparse properties of saddle point problems, direct solution methods are often infeasible. The most suitable approaches are iteration methods, such as preconditioned Krylov subspace methods.

When $\omega=0$, the PDE-constrained optimization problem (1.1)-(1.2) reduces to the Poisson control problem. For this case, there have been several efficient developments on preconditioned iterative techniques using Krylov subspace methods, see [3, 
$4,27,28]$ and the reference therein. In this paper, we deal with the case $\omega \neq 0$. To our knowledge, there are two computational techniques for solving this type of linear systems. The first is the so-called complex-to-real method, i.e., changing the original complex linear system (1.9) to a real one and taking advantages of some real-valued iteration methods, cf., e.g., [6, 10, 13]. This type of methods avoid complex arithmetic operations and may appear to be effective. However, generally four-by-four real block linear systems now need to be solved rather than the original two-bytwo ones, which may increase the computational workload. Indeed, if we denote $y=y_{R}+i y_{I}, y_{d}=y_{d_{R}}+i y_{d_{I}}, \tilde{u}=\widetilde{u}_{R}+i \widetilde{u}_{I}$, and $\widetilde{f}=\widetilde{f}_{R}+i \widetilde{f}_{I}$ then the two-bytwo linear systems (1.8) and (1.9) can be equivalently transformed into the following four-by-four real form:

$$
\left[\begin{array}{cccc}
M & 0 & \sqrt{\beta} K & \sqrt{\beta} \omega M \\
0 & M & -\sqrt{\beta} \omega M & \sqrt{\beta} K \\
\sqrt{\beta} K & -\sqrt{\beta} \omega M & -M & 0 \\
\sqrt{\beta} \omega M & \sqrt{\beta} K & 0 & -M
\end{array}\right]\left[\begin{array}{c}
y_{R} \\
y_{I} \\
\widetilde{u}_{R} \\
\widetilde{u}_{I}
\end{array}\right]=\left[\begin{array}{c}
M y_{d_{R}} \\
M y_{d_{I}} \\
\widetilde{f}_{R} \\
\widetilde{f}_{I}
\end{array}\right]
$$

and

$$
\left[\begin{array}{cccc}
M & 0 & -\sqrt{\beta} K & -\sqrt{\beta} \omega M \\
0 & M & \sqrt{\beta} \omega M & -\sqrt{\beta} K \\
\sqrt{\beta} K & -\sqrt{\beta} \omega M & M & 0 \\
\sqrt{\beta} \omega M & \sqrt{\beta} K & 0 & M
\end{array}\right]\left[\begin{array}{c}
y_{R} \\
y_{I} \\
-\widetilde{u}_{R} \\
-\widetilde{u}_{I}
\end{array}\right]=\left[\begin{array}{c}
M y_{d_{R}} \\
M y_{d_{I}} \\
\widetilde{f}_{R} \\
\widetilde{f}_{I}
\end{array}\right],
$$

respectively. It is noticed that, in Section 2 of [5], under the assumptions that the desired state $y_{d}(x)$ in (1.3) and the computed state $y(x)$ in (1.4) are real-valued, the complex linear system (1.9) is equivalently transformed into a two-by-two real block linear system in [9] and an efficient preconditioner is considered to solve it. In this paper, in contrast to [5], the case of (1.9) with complex valued $y(x)$ and $y_{d}(x)$ is analysed. The alternative method for solving the linear systems (1.8) and (1.9), which is also our choice in this paper, tackles the complex linear system directly. Recently, some special preconditioning and iterative techniques have appeared in the literature to solve the linear system (1.8), see [21, 33, 34]. In this paper, based on the works in $[3,9]$, we construct a new structured two-by-two block preconditioner, which is called $\mathscr{P}_{\text {str-II }}$, for the linear system (1.9). The implementation of $\mathscr{P}_{\text {str-II }}$ is straightforward and the computational complexity is of optimal order. Furthermore, results for the eigenvalue distribution of the corresponding preconditioned matrix show that it leads to a condition number, bounded above by 2 . Numerical results show also that, when the preconditioner $\mathscr{P}_{\text {str-II }}$ is used in a Krylov subspace method, the performance is robust with respect to the related problem parameters.

The paper is organized as follows. In Section 2, the new preconditioner is presented and some existing preconditioning techniques are summarized. In Section 3, implementation details of the new preconditioner $\mathscr{P}_{\text {str-II }}$ are discussed and compared with those of some other preconditioners. In Section 4, the spectral properties of the preconditioned matrix $\mathscr{P}_{\text {str-II }}^{-1} \mathscr{A}_{\text {II }}$ are analyzed. Results on the eigenvalue and eigenvector distributions are presented. In Section 5, numerical comparisons of the performance of the new preconditioner with that of the other preconditioners are given. Finally in Section 6, we end this paper with some concluding remarks. 


\section{The preconditioners}

Due to the Hermitian property of $\mathscr{A}_{1}$, it is suitable to choose the MINRES method [26] to solve (1.8). We know that the fast solution of (1.8) by MINRES requires a good preconditioner, which must be Hermitian positive definite. In [21], Krendl, Simoncini, and Zulehner proposed a block-diagonal preconditioner:

$$
\mathscr{P}_{\text {diag-I }}=\left(\begin{array}{cc}
(1+\sqrt{\beta} \omega) M+\sqrt{\beta} K & 0 \\
0 & (1+\sqrt{\beta} \omega) M+\sqrt{\beta} K
\end{array}\right)
$$

for the coefficient matrix $\mathscr{A}_{\mathrm{I}}$ in (1.8). It is shown that the spectrum of $\mathscr{P}_{\text {diag-I }}^{-1} \mathscr{A}_{\mathrm{I}}$ is contained in the set

$$
\left[-1,-\frac{\sqrt{3}}{3}\right] \cup\left[\frac{\sqrt{3}}{3}, 1\right] .
$$

It should be noted that, by means of the interpolation theory in [35], a preconditioner similar to $\mathscr{P}_{\text {diag-I }}$ is analysed when solving the multiharmonic parabolic optimal control problem [19, 22] and the time-periodic eddy current optimal control problem [20]. More recently in [34], another slightly modified block-diagonal preconditioner is presented:

$$
\mathscr{P}_{\text {diag-II }}=\left(\begin{array}{cc}
\sqrt{1+\beta \omega^{2}} M+\sqrt{\beta} K & 0 \\
0 & \sqrt{1+\beta \omega^{2}} M+\sqrt{\beta} K
\end{array}\right)
$$

for $\mathscr{A}_{\text {I }}$. This preconditioner can result in tighter eigenvalue bounds than $\mathscr{P}_{\text {diag-I. }}$ In fact, it is proved that the spectrum of $\mathscr{P}_{\text {diag-II }}^{-1} \mathscr{A}_{\mathrm{I}}$ is contained in the set

$$
\left[-1,-\frac{\sqrt{2}}{2}\right] \cup\left[\frac{\sqrt{2}}{2}, 1\right] .
$$

Both preconditioners $\mathscr{P}_{\text {diag-I }}$ and $\mathscr{P}_{\text {diag-II }}$ are symmetric positive definite, thus, they can be used within MINRES to solve (1.8). Their efficiency is guaranteed by the tight eigenvalue bounds in (2.2) and (2.4) and a convergence result for the preconditioned MINRES method in [17].

Alternatively, a block-triangular preconditioner is proposed in [34], which is structured as

$$
\mathscr{P}_{\text {tri }}=\left(\begin{array}{cc}
M & 0 \\
\sqrt{\beta}(K+i \omega M) & S
\end{array}\right)
$$

with

$$
S=\left(\sqrt{1+\beta \omega^{2}} M+\sqrt{\beta} K\right) M^{-1}\left(\sqrt{1+\beta \omega^{2}} M+\sqrt{\beta} K\right),
$$

approximating the true Schur complement $\mathscr{S}_{\text {II }}$ of $\mathscr{A}_{\text {II }}, \mathscr{S}_{\text {II }}=\left(1+\beta \omega^{2}\right) M+$ $\beta K M^{-1} K$. This preconditioner can be used to accelerate the performance of nonsymmetric Krylov subspace solvers for solving (1.8). It is proven in [34] that the spectrum of $\mathscr{P}_{\text {tri }}^{-1} \mathscr{A}_{\mathrm{I}}$ is contained in the interval $\left[\frac{1}{2}, 1\right]$. Moreover, in order to optimize the eigenvalue distributions, the two preconditioners $\mathscr{P}_{\text {diag-II }}$ and $\mathscr{P}_{\text {tri }}$ are further generalized in parameter-dependent forms in [34]. See [21, 34, 35] for more details. 
In this paper, for $\mathscr{A}_{\text {II }}$ in (1.9), based on the works in [3, 9], we consider the following structured two-by-two block preconditioner

$$
\mathscr{P}_{\text {str-I }}=\left(\begin{array}{cc}
M & -\sqrt{\beta}(K-i \omega M) \\
\sqrt{\beta}(K+i \omega M) & M+2 \sqrt{\beta} K
\end{array}\right) .
$$

It is shown in $[3,9]$ that the spectrum of $\mathscr{P}_{\text {str-I }}^{-1} \mathscr{A}_{\text {II }}$ is also contained in the interval $\left[\frac{1}{2}, 1\right]$. Moreover, it is proved that the preconditioned matrix $\mathscr{P}_{\text {str-I }}^{-1} \mathscr{A}_{\text {II }}$ is a normal matrix. Thus, it is diagonalizable, which is beneficial to the solution of preconditioned Krylov subspace methods. When $\omega=0$, the numerical experiments in [3,9] show that the performance of the preconditioner $\mathscr{P}_{\text {str-I }}$ within a nonsymmetric solver such as GMRES is robust and totally parameter independent. However, when $\omega \neq 0$, the matrices of the involved systems arising in the implementation of $\mathscr{P}_{\text {str-I }}$ become complex, which increase the computational burden to implement $\mathscr{P}_{\text {str-I. }}$. More details about this preconditioner related to its implementation are discussed in the next section. The main concern of this paper is to overcome this drawback of $\mathscr{P}_{\text {str-I }}$. We construct and analyze the following more efficient structured preconditioner:

$$
\mathscr{P}_{\text {str-II }}=\left(\begin{array}{cc}
M & -\sqrt{\beta}(K-i \omega M) \\
\sqrt{\beta}(K+i \omega M) & M+2 \sqrt{\beta\left(1+\beta \omega^{2}\right)} K
\end{array}\right)
$$

to be applied to $\mathscr{A}_{\text {II }}$ in (1.9). It is obvious that, when $\omega=0$, the preconditioner $\mathscr{P}_{\text {str-II }}$ reduces to the preconditioner $\mathscr{P}_{\text {str-I }}$ in (2.7). Comparing $\mathscr{P}_{\text {str-II }}$ with $\mathscr{P}_{\text {str-I }}$, almost the same eigenvalue distribution result for the preconditioned matrices $\mathscr{P}_{\text {str-I }}^{-1} \mathscr{A}_{\text {II }}$ and $\mathscr{P}_{\text {str-II }}^{-1} \mathscr{A}_{\text {II }}$ can be obtained. However, we show that the implementation of $\mathscr{P}_{\text {str-II }}$ is more computationally cheap, since it does not involve any solutions with complex matrices. Thus, the preconditioner $\mathscr{P}_{\text {str-II }}$ performs much better within nonsymmetric Krylov subspace solvers and can be viewed as an improved modification of the preconditioner $\mathscr{P}_{\text {str-I }}$.

\section{Implementation aspects}

In this section, we first present the algorithmic implementation of the preconditioner $\mathscr{P}_{\text {str-I }}$ and the new preconditioner $\mathscr{P}_{\text {str-II }}$ by using some practical expressions of their inverses. Then we discuss and compare the algorithm to implement $\mathscr{P}_{\text {str-II }}$ with that of $\mathscr{P}_{\text {str-I }}$ and the other preconditioners mentioned in Section 2.

\subsection{Algorithmic implementation of $\mathscr{P}_{\text {str-I }}$}

We first consider the solution of systems with $\mathscr{P}_{\text {str-I }}$ in (2.7). The following linear system needs to be solved:

$$
\mathscr{P}_{\text {str-I }}\left[\begin{array}{l}
z_{1} \\
z_{2}
\end{array}\right]=\left[\begin{array}{l}
r_{1} \\
r_{2}
\end{array}\right], \quad \text { i.e., } \quad\left[\begin{array}{l}
z_{1} \\
z_{2}
\end{array}\right]=\mathscr{P}_{\text {str-I }}^{-1}\left[\begin{array}{l}
r_{1} \\
r_{2}
\end{array}\right] \text {. }
$$


As derived in $[3,9]$, the exact inverse of $\mathscr{P}_{\text {str-I }}$ is as follows:

$$
\mathscr{P}_{\text {str-I }}^{-1}=\left[\begin{array}{cc}
H_{1}^{-1}+H_{2}^{-1}-H_{2}^{-1} M H_{1}^{-1} & \left(I-H_{2}^{-1} M\right) H_{1}^{-1} \\
-H_{2}^{-1}\left(I-M H_{1}^{-1}\right) & H_{2}^{-1} M \tilde{H}_{1}^{-1}
\end{array}\right]
$$

with

$$
H_{1}=(M+\sqrt{\beta} K)+i \omega \sqrt{\beta} M \quad \text { and } \quad H_{2}=(M+\sqrt{\beta} K)-i \omega \sqrt{\beta} M .
$$

Then the action of $\mathscr{P}_{\text {str-I }}^{-1}$ on a vector is implemented in the following steps:

Algorithm 31 Computing the solution $z$ of $\mathscr{P}_{\text {str-II }} z=r$ with $z=\left[z_{1}, z_{2}\right]^{T}$ and $r=\left[r_{1}, r_{2}\right]^{T}$ :

1. Solve $H_{1} g=r_{1}+r_{2}$;

2. Solve $H_{2} z_{2}=M g-r_{1}$;

3. Compute $z_{1}=g-z_{2}$.

We see, that two complex symmetric linear systems with coefficient matrices defined as (3.3) need to be solved at step (1) and step (2) of Algorithm 31. If we denote

$$
r_{1}=r_{1_{R}}+i r_{1_{I}}, \quad r_{2}=r_{2_{R}}+i r_{2_{I}}, \quad g=g_{R}+i g_{I} \quad \text { and } \quad z_{2}=z_{R}+i z_{I},
$$

then, based on the idea of solving complex valued systems in real arithmetic $[6,13]$, these complex linear systems can equivalently be transformed into the following real forms:

$$
\begin{aligned}
& {\left[\begin{array}{cc}
M+\sqrt{\beta} K & -\omega \sqrt{\beta} M \\
\omega \sqrt{\beta} M & M+\sqrt{\beta} K
\end{array}\right]\left[\begin{array}{c}
g_{R} \\
g_{I}
\end{array}\right]=\left[\begin{array}{c}
r_{1_{R}}+r_{2_{R}} \\
r_{1_{I}}+r_{2_{I}}
\end{array}\right] \text { and }} \\
& {\left[\begin{array}{cc}
M+\sqrt{\beta} K & \omega \sqrt{\beta} M \\
-\omega \sqrt{\beta} M & M+\sqrt{\beta} K
\end{array}\right]\left[\begin{array}{c}
z_{R} \\
z_{I}
\end{array}\right]=\left[\begin{array}{c}
M g_{R}-r_{1_{R}} \\
M g_{I}-r_{1_{I}}
\end{array}\right],}
\end{aligned}
$$

respectively. It follows from [9] that the above two linear systems can be solved by GMRES with the following robust and efficient preconditioners:

$$
\begin{aligned}
& \mathscr{P}_{1}=\left[\begin{array}{cc}
M+\sqrt{\beta} K & -\omega \sqrt{\beta} M \\
\omega \sqrt{\beta} M & M+\sqrt{\beta} K+2 \omega \sqrt{\beta} K
\end{array}\right] \text { and } \\
& \mathscr{P}_{2}=\left[\begin{array}{cc}
M+\sqrt{\beta} K & \omega \sqrt{\beta} M \\
-\omega \sqrt{\beta} M & M+\sqrt{\beta} K+2 \omega \sqrt{\beta} K
\end{array}\right] .
\end{aligned}
$$

For applying the preconditioners $\mathscr{P}_{1}$ and $\mathscr{P}_{2}$, we follow the strategy in [5]. In fact, $\mathscr{P}_{1}$ and $\mathscr{P}_{2}$ can be factorized as

$$
\mathscr{P}_{1}=\left[\begin{array}{cc}
I & -I \\
0 & I
\end{array}\right]\left[\begin{array}{cc}
(1+\sqrt{\beta} \omega) M+\sqrt{\beta} K & 0 \\
\omega \sqrt{\beta} M & (1+\sqrt{\beta} \omega) M+\sqrt{\beta} K
\end{array}\right]\left[\begin{array}{ll}
I & I \\
0 & I
\end{array}\right]
$$

and

$$
\mathscr{P}_{2}=\left[\begin{array}{cc}
I & I \\
0 & I
\end{array}\right]\left[\begin{array}{cc}
(1+\sqrt{\beta} \omega) M+\sqrt{\beta} K & 0 \\
-\omega \sqrt{\beta} M & (1+\sqrt{\beta} \omega) M+\sqrt{\beta} K
\end{array}\right]\left[\begin{array}{cc}
I & -I \\
0 & I
\end{array}\right] .
$$


Then we obtain the following algorithms:

Algorithm 32 Solve $\mathscr{P}_{1} z=r$ with $z=\left[z_{1}, z_{2}\right]^{T}$ and $r=\left[r_{1}, r_{2}\right]^{T}$ :

1. Solve $((1+\sqrt{\beta} \omega) M+\sqrt{\beta} K) g_{1}=r_{1}+r_{2}$;

2. Solve $((1+\sqrt{\beta} \omega) M+\sqrt{\beta} K) z_{2}=r_{2}-\omega \sqrt{\beta} M g_{1}$;

3. Compute $z_{1}=g_{1}-z_{2}$.

Algorithm 33 Solve $\mathscr{P}_{2} z=r$ with $z=\left[z_{1}, z_{2}\right]^{T}$ and $r=\left[r_{1}, r_{2}\right]^{T}$ :

1. Solve $((1+\sqrt{\beta} \omega) M+\sqrt{\beta} K) g_{2}=r_{1}-r_{2}$;

2. Solve $((1+\sqrt{\beta} \omega) M+\sqrt{\beta} K) z_{2}=r_{2}+\omega \sqrt{\beta} M g_{2}$;

3. Compute $z_{1}=g_{2}+z_{2}$.

As stated in [5], the above two algorithms are more efficient compared with those in [9] to implement $\mathscr{P}_{1}$ and $\mathscr{P}_{2}$, which are based on their inverse expressions similar to (3.2). The major differences are that in Algorithm 32 and Algorithm 33, we perform a matrix-vector multiplication with $M$ rather than $M+\sqrt{\beta} K$, which is obviously much easier to handle. See [5, 9] for more details. Now, combining Algorithm 31 with Algorithm 32 and Algorithm 33, the implementation of $\mathscr{P}$ str-I within a Krylov subspace solver can be implemented as an inner-outer iterative procedure.

\subsection{Algorithmic implementation of $\mathscr{P}_{\text {str-II }}$}

We show next an explicit form of the inverse of $\mathscr{P}_{\text {str-II }}$ using the block matrix factorization technique in $[2,8]$ for a two-by-two block matrix.

Theorem 34 Assume that $M$ and $K$ are symmetric positive definite matrices. Then, the inverse of the preconditioner $\mathscr{P}_{\text {str-II }}$ in (2.8), is of the form:

$$
\mathscr{P}_{\text {str-II }}^{-1}=\left[\begin{array}{cc}
\left(\beta_{\omega_{1}}+\beta_{\omega_{2}}\right) H^{-1}-\beta_{\omega_{1}} \beta_{\omega_{2}} H^{-1} M H^{-1} & \left(I-\beta_{\omega_{2}} H^{-1} M\right) H^{-1} \\
-H^{-1}\left(I-\beta_{\omega_{1}} M H^{-1}\right) & H^{-1} M H^{-1}
\end{array}\right]
$$

with

$$
\beta_{\omega_{1}}=\sqrt{1+\beta \omega^{2}}-i \sqrt{\beta} \omega \text { and } \beta_{\omega_{2}}=\sqrt{1+\beta \omega^{2}}+i \sqrt{\beta} \omega
$$

and

$$
H=\sqrt{1+\beta \omega^{2}} M+\sqrt{\beta} K
$$

Proof Note that $\mathscr{P}_{\text {str-II }}$ can be factorized as

$$
\mathscr{P}_{\text {str-II }}=\left[\begin{array}{cc}
M & 0 \\
\sqrt{\beta}(K+i \omega M) & S
\end{array}\right]\left[\begin{array}{cc}
I-\sqrt{\beta} M^{-1}(K-i \omega M) \\
0
\end{array}\right],
$$

where the Schur complement matrix $S$ is defined in (2.6). Then, based on (3.7), it is straightforward to verify that

$$
\mathscr{P}_{\text {str-II }}^{-1}=\left[\begin{array}{cc}
I & \sqrt{\beta} M^{-1}(K-i \omega M) \\
0 & I
\end{array}\right]\left[\begin{array}{cc}
M^{-1} & 0 \\
-\sqrt{\beta} S^{-1}(K+i \omega M) M^{-1} & S^{-1}
\end{array}\right] .
$$


A simple calculation leads to

$$
\mathscr{P}_{\text {str-II }}^{-1}=\left[\begin{array}{cc}
M^{-1}-\beta M^{-1}(K-i \omega M) S^{-1}(K+i \omega M) M^{-1} & \sqrt{\beta} M^{-1}(K-i \omega M) S^{-1} \\
-\sqrt{\beta} S^{-1}(K+i \omega M) M^{-1} & S^{-1}
\end{array}\right] .
$$

With the notations in (2.6), (3.5), and (3.6), we have $S^{-1}=H^{-1} M H^{-1}$ and

$$
\begin{aligned}
M^{-1}-\beta M^{-1}(K-i \omega M) S^{-1}(K+i \omega M) M^{-1} & =M^{-1}-M^{-1}\left(H-\beta_{\omega_{2}} M\right) H^{-1} M H^{-1}\left(I-\beta_{\omega_{1}} H^{-1} M\right) M^{-1}, \\
\sqrt{\beta} M^{-1}(K-i \omega M) S^{-1} & =M^{-1}\left(H-\beta_{\omega_{2}} M\right) H^{-1} M H^{-1}, \\
\sqrt{\beta} S^{-1}(K+i \omega M) M^{-1} & =H^{-1} M H^{-1}\left(H-\beta_{\omega_{1}} M\right) M^{-1} .
\end{aligned}
$$

Combining with (3.8), we obtain the form of the inverse in (3.4).

Having an explicit form of the inverse of $\mathscr{P}_{\text {str-II }}$, we are now in a position to derive an efficient implementation of the action of $\mathscr{P}_{\text {str-II }}^{-1}$ on a vector, namely,

$$
\begin{aligned}
\mathscr{P}_{\text {str-II }}^{-1}\left[\begin{array}{l}
r_{1} \\
r_{2}
\end{array}\right] & =\left[\begin{array}{c}
H^{-1}\left(\beta_{\omega_{1}} r_{1}+r_{2}\right)+\beta_{\omega_{2}} H^{-1} r_{1}-\beta_{\omega_{2}} H^{-1} M H^{-1}\left(\beta_{\omega_{1}} r_{1}+r_{2}\right) \\
-H^{-1} r_{1}+H^{-1} M H^{-1}\left(\beta_{\omega_{1}} r_{1}+r_{2}\right)
\end{array}\right] \\
& =\left[\begin{array}{c}
g+\beta_{\omega_{2}} H^{-1} r_{1}-\beta_{\omega_{2}} H^{-1} M g \\
-H^{-1} r_{1}+H^{-1} M g
\end{array}\right] \\
& =\left[\begin{array}{c}
g-\beta_{\omega_{2}} H^{-1}\left(M g-r_{1}\right) \\
H^{-1}\left(M g-r_{1}\right)
\end{array}\right],
\end{aligned}
$$

where $g=H^{-1}\left(\beta_{\omega_{1}} r_{1}+r_{2}\right)$. In an algorithmic form,

Algorithm 35 Solve $\mathscr{P}_{\text {str-II }} z=r$ with $z=\left[z_{1}, z_{2}\right]^{T}$ and $r=\left[r_{1}, r_{2}\right]^{T}$

1. Solve $\left(\sqrt{1+\beta \omega^{2}} M+\sqrt{\beta} K\right) g=\left(\sqrt{1+\beta \omega^{2}}-i \sqrt{\beta} \omega\right) r_{1}+r_{2}$;

2. Solve $\left(\sqrt{1+\beta \omega^{2}} M+\sqrt{\beta} K\right) z_{2}=M g-r_{1}$;

3. Compute $z_{1}=g-\left(\sqrt{1+\beta \omega^{2}}+i \sqrt{\beta} \omega\right) z_{2}$.

\subsection{Comparisons between the mentioned preconditioners}

Next, we present the computational cost to apply the preconditioner $\mathscr{P}_{\text {str-II }}$, compared with that of the other preconditioners, defined in Section 2.

- Compared with the implementations of the two block-diagonal preconditioners $\mathscr{P}_{\text {diag-I }}, \mathscr{P}_{\text {diag-II }}$, it is evident that the workload for the related algorithms is almost the same except for one more matrix-vector multiplication in step (2) of Algorithm 35. However, it is shown in the next section that the preconditioned matrix $\mathscr{P}_{\text {str-II }}^{-1} \mathscr{A}$ has tighter eigenvalue distribution, which entails much fewer iteration steps.

- The block-triangular preconditioner $\mathscr{P}_{\text {tri }}$ requires one more solution of a system with the matrix $M$ and two more matrix and vector multiplication with $M$ and $K$. In the next section, we show that $\mathscr{P}_{\text {str-II }}^{-1} \mathscr{A}$ and $\mathscr{P}_{\text {tri }}^{-1} \mathscr{A}$ have the same eigenvalue distribution results.

- Comparing the computational procedure for the preconditioner $\mathscr{P}_{\text {str-I }}$ in Algorithm 31 with that in Algorithm 32 and Algorithm 33, we see that, even ignoring 
the extra vector additions, $\mathscr{P}_{\text {str-I }}$ requires two more solutions of systems with the coefficient matrix $(1+\sqrt{\beta} \omega) M+\beta K$. Moreover, in the next section, we show that, up to a lower bound, the eigenvalues of the preconditioned matrices $\mathscr{P}_{\text {str-I }}^{-1} \mathscr{A}$ and $\mathscr{P}_{\text {str-II }}^{-1} \mathscr{A}$ are located in the same interval, so it is not possible to compensate the higher computational cost by faster convergence.

Thus, from implementation point of view, the preconditioner $\mathscr{P}_{\text {str-II }}$ is most efficient compared with the other considered preconditioners.

It is worth mentioning that, in each of the algorithms mentioned above, solutions with either systems with the matrix $(1+\sqrt{\beta} \omega) M+\sqrt{\beta} K$ or systems with the matrix $\sqrt{1+\beta \omega^{2}} M+\sqrt{\beta} K$ appear. The two matrices have the same structure up to a minor difference between the coefficient in front of $M$. Note that

$$
\lim _{\beta \rightarrow 0} \frac{1+\sqrt{\beta} \omega}{\sqrt{1+\beta \omega^{2}}}=1,
$$

thus, the two matrices are almost the same when $\beta$ approaches 0 . So we can expect that the workload for solving the related systems are almost the same.

\section{Spectral properties}

For completeness, we first analyze the eigenvalue distribution of the preconditioned matrix $\mathscr{P}_{\text {str-I }}^{-1} \mathscr{A}_{\text {III }}$. The obtained result is a supplement of the results in [3,9].

Theorem 41 Assume that $M$ and $K$ are symmetric positive definite matrices. Then the eigenvalues $\lambda$ of the preconditioned matrix $\mathscr{P}_{\text {str-I }}^{-1} \mathscr{A}_{I I}$ are either equal to 1 or are of the form

$$
\frac{1+\beta\left(1+\omega^{2}\right) \xi^{2}}{1+\beta\left(1+\omega^{2}\right) \xi^{2}+2 \sqrt{\beta} \xi} \in\left(\frac{1}{2}, 1\right),
$$

where $\xi>0$ is an eigenvalue of the matrix $M^{-1} K$. Also there holds that

$$
\lambda \in\left(\frac{1}{2}, 1\right],
$$

independently of the parameters $h, \beta$ and $\omega$.

Proof Consider the matrix splitting $\mathscr{A}_{\text {II }}=\mathscr{P}_{\text {str-I }}-\mathscr{R}_{\text {str-I }}$ with

$$
\mathscr{R}_{\text {str-I }}=\left[\begin{array}{cc}
0 & 0 \\
0 & 2 \sqrt{\beta} K
\end{array}\right],
$$

then it follows from (3.2) that

$$
\mathscr{P}_{\text {str-I }}^{-1} \mathscr{A}_{\text {II }}=I-\mathscr{P}_{\text {str-I }}^{-1} \mathscr{R}_{\text {str-I }}=\left[\begin{array}{cc}
I-2 \sqrt{\beta}\left(I-H_{2}^{-1} M\right) H_{1}^{-1} K \\
0 & I-2 \sqrt{\beta} H_{2}^{-1} M H_{1}^{-1} K
\end{array}\right]
$$

with $H_{1}$ and $H_{2}$ defined as (3.3). It is straightforward to verify that

$$
H_{1} M^{-1} H_{2}=M+\beta\left(1+\omega^{2}\right) K M^{-1} K+2 \sqrt{\beta} K,
$$

which leads to 


$$
I-2 \sqrt{\beta} H_{2}^{-1} M H_{1}^{-1} K=\left(M+\beta\left(1+\omega^{2}\right) K M^{-1} K+2 \sqrt{\beta} K\right)^{-1}\left(M+\beta\left(1+\omega^{2}\right) K M^{-1} K\right) .
$$

Assume that $\{\mu, u\}$ is an eigenpair of $I-2 \sqrt{\beta} H_{2}^{-1} M H_{1}^{-1} K$. Then, based on (4.4), it holds that

$$
\left(M+\beta\left(1+\omega^{2}\right) K M^{-1} K\right) u=\mu\left(M+\beta\left(1+\omega^{2}\right) K M^{-1} K+2 \sqrt{\beta} K\right) u .
$$

Multiplying the above equation by $M^{-\frac{1}{2}}$ from both sides, we have

$$
\left(I+\beta\left(1+\omega^{2}\right) \widehat{K}^{2}\right) \widehat{u}=\mu\left(I+\beta\left(1+\omega^{2}\right) \widehat{K}^{2}+2 \sqrt{\beta} \widehat{K}\right) \widehat{u}
$$

with $\widehat{K}=M^{-\frac{1}{2}} K M^{-\frac{1}{2}}$ and $\widehat{u}=M^{\frac{1}{2}} u$. Then, utilizing that the symmetric positive definite matrix $\widehat{K}$ is similar to $M^{-1} K$, we obtain the eigenvalue distribution result in (4.1). Here, we have used the fact that

$$
\mu=\frac{1+\beta\left(1+\omega^{2}\right) \xi^{2}}{1+\beta\left(1+\omega^{2}\right) \xi^{2}+2 \sqrt{\beta} \xi}>\frac{1+\beta\left(1+\omega^{2}\right) \xi^{2}}{1+\beta\left(1+\omega^{2}\right) \xi^{2}+2 \sqrt{\beta\left(1+\omega^{2}\right)} \xi}=\frac{1+\widehat{\xi}^{2}}{(1+\widehat{\xi})^{2}}
$$

with $\widehat{\xi}=\sqrt{\beta\left(1+\omega^{2}\right)} \xi$. Then, based on (4.1) and (4.3), we obtain the eigenvalue distribution result in (4.2).

Remark 42 Under the assumption that both $K$ and $M$ are symmetric positive definite and $\omega>0$, the obtained eigenvalue bound $\left(\frac{1}{2}, 1\right]$ in $(4.1)$ is tighter than that in $[3,9]$, i.e., $\left[\frac{1}{2}, 1\right]$, for $\mathscr{P}_{\text {str-I }}^{-1} \mathscr{A}_{\text {III }}$. Further, if $\beta \omega^{2} \xi^{2} \gg 1$, all eigenvalues are tightly clustered near the unit value.

Now we analyze the spectrum of the preconditioned matrix $\mathscr{P}_{\text {str-II }}^{-1} \mathscr{A}_{\text {II }}$. The following lemma is useful.

Lemma 43 Assume that $M$ and $K$ are symmetric positive definite matrices, $\mathscr{S}_{I I}=$ $\left(1+\beta \omega^{2}\right) M+\beta K M^{-1} K$ and $S$ is defined in (2.6). Then each eigenvalue $\mu$ of $S^{-1} \mathscr{S}_{I I}$ satisfies

$$
\mu=\frac{1+\xi^{2}}{(1+\xi)^{2}} \in\left[\frac{1}{2}, 1\right),
$$

where $\xi>0$ is an eigenvalue of the matrix $\sqrt{\gamma} M^{-1} K$ with $\gamma=\frac{\beta}{1+\beta \omega^{2}}$.

Proof Assume that $\{\mu, u\}$ is an eigenpair of $S^{-1} \mathscr{S}_{\text {II }}$, thus

$$
\mathscr{S}_{\mathrm{II}} u=\mu S u \quad \text { with } \quad u \neq 0 .
$$

Then, it is obvious that $\mu>0$ as $\mathscr{S}_{\text {II }}$ and $S$ are both symmetric positive definite matrices. Based on

$$
\left(\left(1+\beta \omega^{2}\right) M+\beta K M^{-1} K\right) u=\mu\left(\left(1+\beta \omega^{2}\right) M+\beta K M^{-1} K+2 \sqrt{\beta\left(1+\beta \omega^{2}\right)} K\right) u
$$

and the fact that $M$ is symmetric positive definite, we obtain the equality

$$
\left(I+\gamma M^{-1} K M^{-1} K\right) u=\mu\left(I+\sqrt{\gamma} M^{-1} K\right)^{2} u .
$$


After multiplying by $M^{-\frac{1}{2}}$ from both sides we obtain

$$
\left(I+\widetilde{K}^{2}\right) \widetilde{u}=\mu(I+\widetilde{K})^{2} \widetilde{u}
$$

with $\widetilde{K}=\sqrt{\gamma} M^{-\frac{1}{2}} K M^{-\frac{1}{2}}$ and $\tilde{u}=M^{\frac{1}{2}} u$. Then, since $\widetilde{K}$ is symmetric positive definite, we have

$$
\mu=\frac{1+\xi^{2}}{(1+\xi)^{2}}
$$

with $\xi>0$ being an eigenvalue of $\widetilde{K}$. Since $\sqrt{\gamma} M^{-1} K$ is similar to $\widetilde{K}$, we can obtain the result in (4.5) by considering the monotonicity of $\mu$ with respective to $\xi$. (It suffices that $K$ is spsd.)

Then, based on Lemma 43, the following eigenvalue bounds for $\mathscr{P}_{\text {str-II }}^{-1} \mathscr{A}_{\text {II }}$ hold true.

Theorem 44 Assume that $M$ and $K$ are symmetric positive definite matrices. Then the eigenvalue $\lambda$ of the preconditioned matrix $\mathscr{P}_{\text {str-II }}^{-1} \mathscr{A}_{I I}$, which is either equal to 1 or of the form (4.5), lies in the interval

$$
\lambda \in\left[\frac{1}{2}, 1\right],
$$

independently of the parameters $h, \beta$ and $\omega$.

Proof Analogously to the proof of Theorem 41, based on (3.4), we have

$$
\mathscr{P}_{\text {str-II }}^{-1} \mathscr{A}_{\mathrm{II}}=\left[\begin{array}{c}
I-2 \sqrt{\beta\left(1+\beta \omega^{2}\right)}\left(I-\left(\sqrt{1+\beta \omega^{2}}+i \sqrt{\beta} \omega\right) H^{-1} M\right) H^{-1} K \\
0 \\
I-2 \sqrt{\beta\left(1+\beta \omega^{2}\right)} H^{-1} M H^{-1} K
\end{array}\right]
$$

with $H$ defined as (3.6). In fact,

$I-2 \sqrt{\beta\left(1+\beta \omega^{2}\right)} H^{-1} M H^{-1} K=H^{-1} M H^{-1}\left(H M^{-1} H-2 \sqrt{\beta\left(1+\beta \omega^{2}\right)} K\right)=S^{-1} \mathscr{S}_{\text {II }}$

with $\mathscr{S}_{\text {II }}=\left(1+\beta \omega^{2}\right) M+\beta K M^{-1} K$ being the Schur complement of $\mathscr{A}_{\text {II }}$ and $S$ being defined as (2.6). From Lemma 43, we know that the eigenvalues of $S^{-1} \mathscr{S}_{\text {II }}$ are located in the interval $\mu \in\left[\frac{1}{2}, 1\right)$. Then, combining with (4.7), we obtain the eigenvalue distribution result in (4.6).

Remark 45 Compared with the related results in [21, 34], it is evident that the spectral bounds for $\mathscr{P}_{\text {str-II }}^{-1} \mathscr{A}_{\text {II }}$ in (4.6) are much better than those in (2.2) and (2.4) for $\mathscr{P}_{\text {diag-I }}^{-1} \mathscr{A}_{\mathrm{I}}$ and $\mathscr{P}_{\text {diag-I }}^{-1} \mathscr{A}_{\mathrm{I}}$. Moreover, it is indicated in [34] that the eigenvalues of the preconditioned matrix $\mathscr{P}_{\text {tri }}^{-1} \mathscr{A}_{\mathrm{I}}$ are also located in the same interval $\left[\frac{1}{2}, 1\right]$ for $\mathscr{P}_{\text {str-II }}^{-1} \mathscr{A}_{\text {III }}$. Furthermore, a comparison of the results in Theorem 41 and Theorem 44 shows that the eigenvalues of the preconditioned matrix $\mathscr{P}_{\text {str-II }}^{-1} \mathscr{A}_{\text {II }}$ and the preconditioned matrix $\mathscr{P}_{\text {str-I }}^{-1} \mathscr{A}_{\text {II }}$ are located in the same interval except for a small variation of the lower bound. However, as we show, the action of $\mathscr{P}_{\text {str-II }}$ is computationally much cheaper than that of $\mathscr{P}_{\text {str-I }}$ and $\mathscr{P}_{\text {tri }}$. 
Next, we analyze the properties of the eigenvector distribution of the matrix $\mathscr{P}_{\text {str-II }}^{-1} \mathscr{A}_{\text {II }}$.

Theorem 46 Assume that $M \in \mathbb{R}^{n \times n}$ and $K \in \mathbb{R}^{n \times n}$ are symmetric positive definite matrices and $H$ is defined as (3.6). Then the preconditioned matrix $\mathscr{P}_{\text {str-II }}^{-1} \mathscr{A}_{I I}$ has $2 n$ linearly independent eigenvectors, which are

1. $n$ eigenvectors of the form $\left[u^{T} 0^{T}\right]^{T}$, that correspond to the eigenvalue 1 ;

2. $n$ eigenvectors of the form $\left[\begin{array}{ll}u^{T} & v^{T}\end{array}\right]^{T}$, with $u=\frac{2 \beta \sqrt{1+\beta \omega^{2}}}{1-\lambda} H^{-1}(K-$ $i \omega M) H^{-1} K v$ and $\mathscr{S}_{I I} v=\lambda S v$ that correspond to the rest of the eigenvalues.

Proof Assume that $\lambda$ and $\left[u^{T} v^{T}\right]^{T}$ with $u, v \in \mathbb{C}^{n}$ is an eigenpair of $\mathscr{P}_{\text {str-II }}^{-1} \mathscr{A}_{\text {II }}$. Then, based on (4.7), we have

$$
\left[\begin{array}{cc}
I-2 \sqrt{\beta\left(1+\beta \omega^{2}\right)}\left(I-\left(\sqrt{1+\beta \omega^{2}}+i \sqrt{\beta} \omega\right) H^{-1} M\right) H^{-1} K \\
0 \\
S^{-1} \mathscr{S}_{\mathrm{II}}
\end{array}\right]\left[\begin{array}{l}
u \\
v
\end{array}\right]=\lambda\left[\begin{array}{l}
u \\
v
\end{array}\right],
$$

where $\mathscr{S}_{\text {II }}=\left(1+\beta \omega^{2}\right) M+\beta K M^{-1} K$ and $S=H M^{-1} H$. It then follows that $u-2 \sqrt{\beta\left(1+\beta \omega^{2}\right)}\left(\left(I-\left(\sqrt{1+\beta \omega^{2}}+i \sqrt{\beta} \omega\right) H^{-1} M\right) H^{-1} K\right) v=\lambda u$ and $\mathscr{S}_{\mathrm{II}} v=\lambda S v$.

Now we consider the following two cases:

1. If $v=0$, from the first equation of (4.8), we have $\lambda=1$. Otherwise, it leads to $u=0$, which contradicts with the requirement that the eigenvectors are nontrivial. Thus we obtain the result in (1);

2. If $v \neq 0$, it must hold that $\lambda \neq 1$. Otherwise, (4.8) reduces to

$$
2 \sqrt{\beta\left(1+\beta \omega^{2}\right)}\left(\left(I-\left(\sqrt{1+\beta \omega^{2}}+i \sqrt{\beta} \omega\right) H^{-1} M\right) H^{-1} K\right) v=0 \text { and } \mathscr{S}_{\mathrm{II}} v=S v \text {. }
$$

Then, from the second equation of (4.9), we have $K v=0$. Considering the positive definiteness of $K$, it follows that $v=0$, which contradicts the assumption that $v \neq 0$. Since $\lambda \neq 1$, it is easy to obtain the result in (2).

Remark 47 Analogously to the discussions in [3], utilizing the symmetric positive definiteness of $S$, we have seen that $S^{-1} \mathscr{S}_{\text {II }}$ can be symmetrized by the similarity transformation $S^{-1} \mathscr{S}_{\text {II }}=S^{-\frac{1}{2}} \mathscr{S}_{\text {II }} S^{-\frac{1}{2}}$. Hence, $S^{-1} \mathscr{S}_{\text {II }}$ has a complete eigenvector space. It then follows that $\mathscr{P}_{\text {str-II }}^{-1} \mathscr{A}_{\text {II }}$ is a normal matrix and diagonalizable, which is beneficial in the Krylov subspace iteration framework.

\section{Numerical experiments}

In this section, we test the numerical performance of the new preconditioner $\mathscr{P}_{\text {str-II }}$ and compare it with that of the preconditioners $\mathscr{P}_{\text {diag-I }}, \mathscr{P}_{\text {diag-II }}, \mathscr{P}_{\text {tri }}$, and $\mathscr{P}_{\text {str-I }}$. The 
Table 1 Comparison of the performance of the preconditioners, $h=\frac{1}{64}$

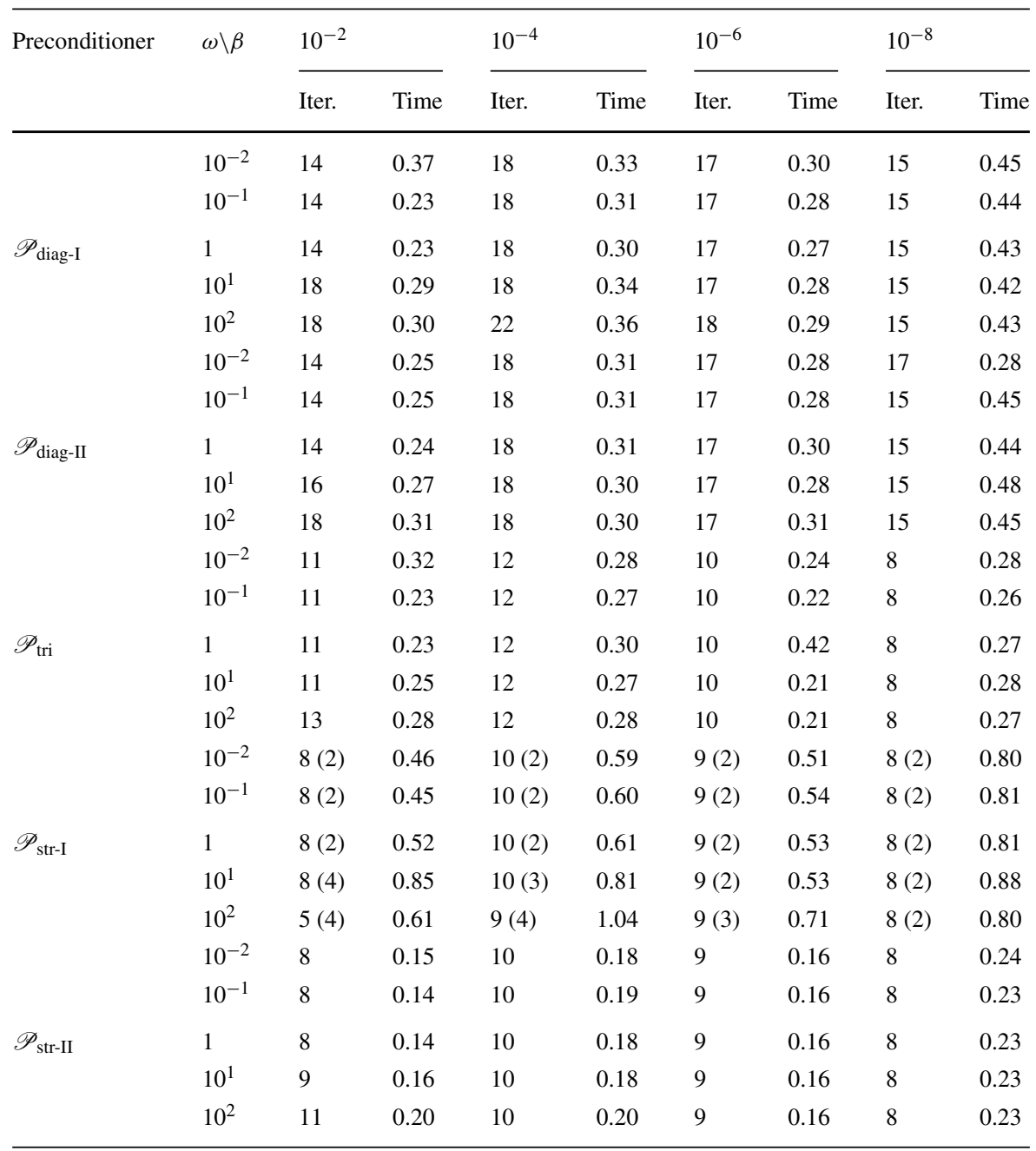

test problem we consider is given by (1.1) and (1.2) with $k=1, \Omega=[0,1]^{2}$ and zero Dirichlet boundary condition $f(x, t)=0$ on $\Sigma_{T}$. The desired state is as in (1.3),

$$
y_{d}(x)=\left\{\begin{array}{cc}
\left(2 x_{1}-1\right)^{2}\left(2 x_{2}-1\right)^{2}, & \text { if }\left(x_{1}, x_{2}\right) \in\left(0, \frac{1}{2}\right)^{2}, \\
0, & \text { otherwise. }
\end{array}\right.
$$

We choose the bilinear quadrilateral finite element $Q_{1}$ to discretize the state, the control and the Lagrangian multiplier on uniform grid with meshsize $h$. In our implementations, the relevant finite element matrices are constructed using the IFISS software package $[15,16]$. To solve the linear system $(1.8)$, we use either MINRES, 
Table 2 Comparison of the performance of the preconditioners, $h=\frac{1}{128}$

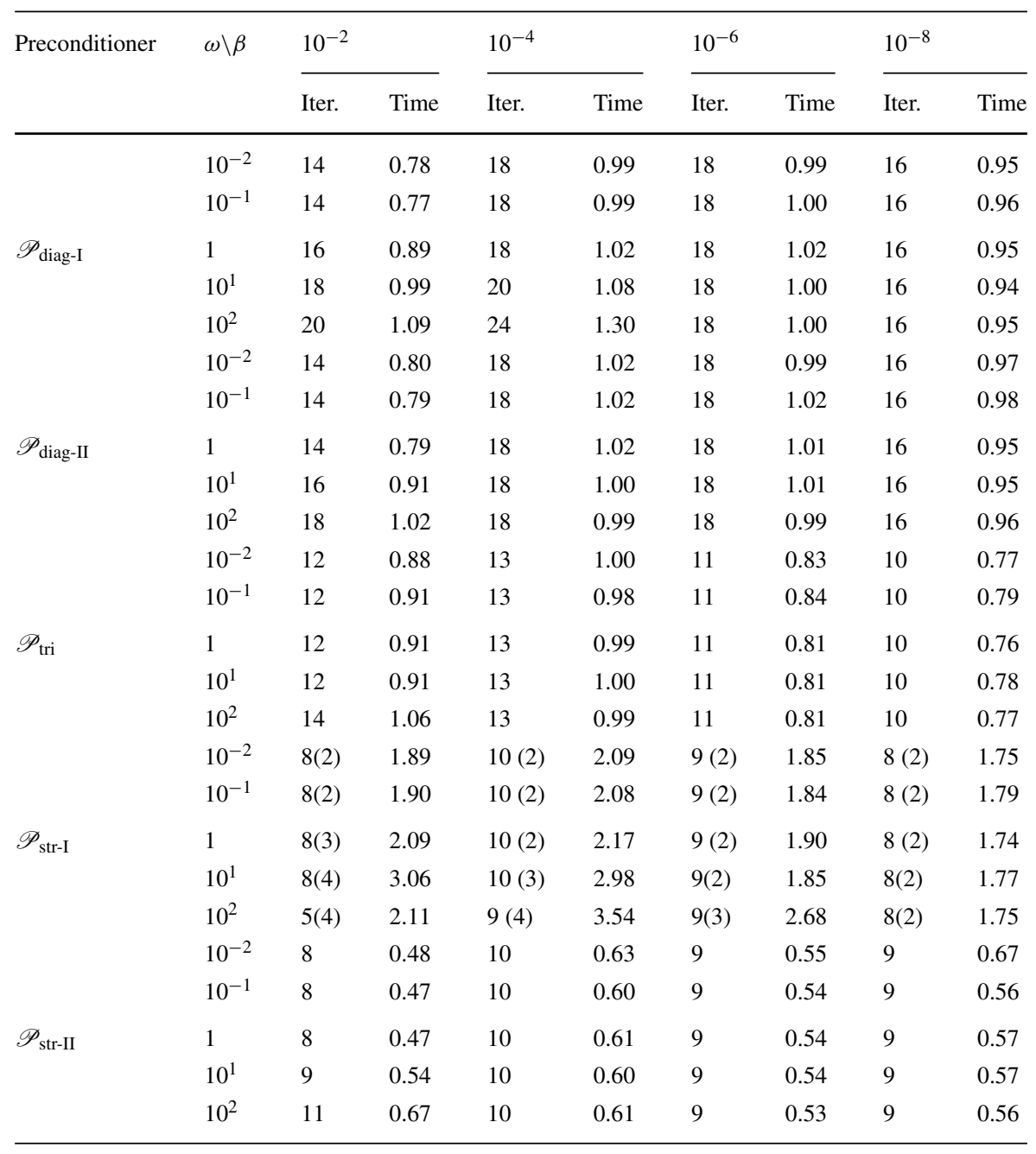

preconditioned by $\mathscr{P}_{\text {diag-I }}$ and $\mathscr{P}_{\text {diag-II }}$, or GMRES, preconditioned by $\mathscr{P}_{\text {tri }}$. The linear system (1.9) is solved by GMRES, preconditioned using $\mathscr{P}_{\text {str-I }}$ and $\mathscr{P}_{\text {str-II }}$. When using $\mathscr{P}_{\text {str-I, }}$ an inner-outer solution framework is employed, as discussed in Section 3. The stopping tolerance for the outer MINRES or GMRES method is set to $10^{-6}$. For the $\mathscr{P}_{\text {str-I }}$ preconditioned GMRES method, the stopping tolerance for the inner GMRES method is set to $10^{-3}$. As for the solution of the arising systems with matrices

$$
(1+\sqrt{\beta} \omega) M+\sqrt{\beta} K \text { or } \sqrt{1+\beta \omega^{2}} M+\sqrt{\beta} K,
$$

we use the default AMG smoothing strategy of the IFISS software package, i.e., the coefficient matrix is approximated by the action of two AMG V-cycles with two 
Table 3 Comparison of the performance of the preconditioners, $h=\frac{1}{256}$

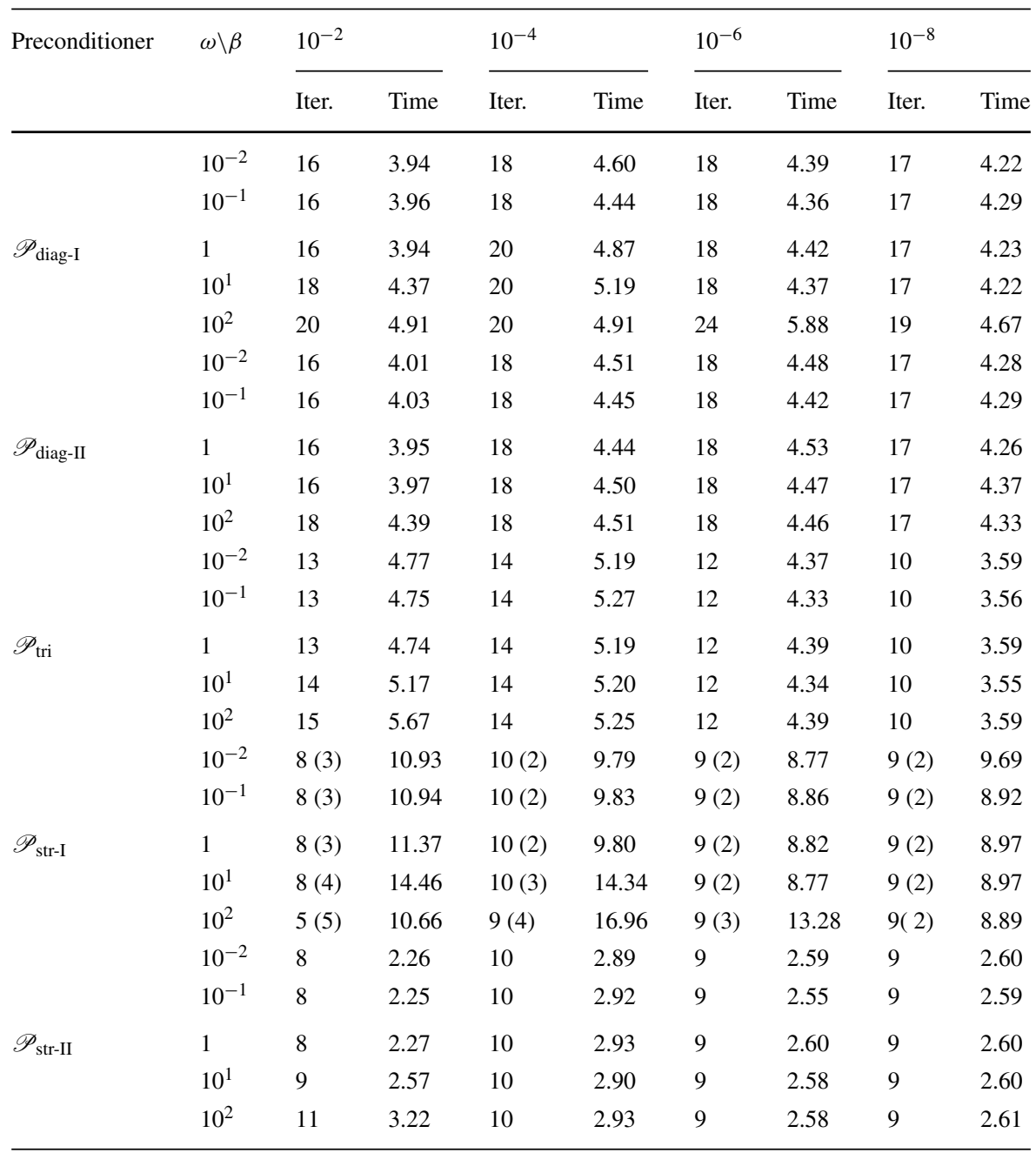

presmoothing and postsmoothing point-damped Jacobi iterations. When the preconditioner $\mathscr{P}_{\text {tri }}$ is applied, the solution of the arising systems with the matrix $M$ is approximated by ten steps of the Chebyshev semi-iteration method.

The numerical results of the tested methods for different mesh sizes $h$, frequency $\omega$ and regularization parameter $\beta$, are depicted in Tables $1,2,3$, and 4 . In all the tables, for each value of $h, \beta$, and $\omega$, we present the number of outer MINRES or GMRES iterations followed by the elapsed CPU time in seconds. In particular, for the $\mathscr{P}_{\text {str-I }}$ preconditioned GMRES method, the adjacent brackets after the outer GMRES iteration steps show the average inner GMRES iterations for each outer iteration.

From Tables 1-4 we observe that, generally, the performance of all the preconditioners is robust with respect to the involved parameters. In accordance with the 
Table 4 Comparison of the performance of the preconditioners, $h=\frac{1}{512}$

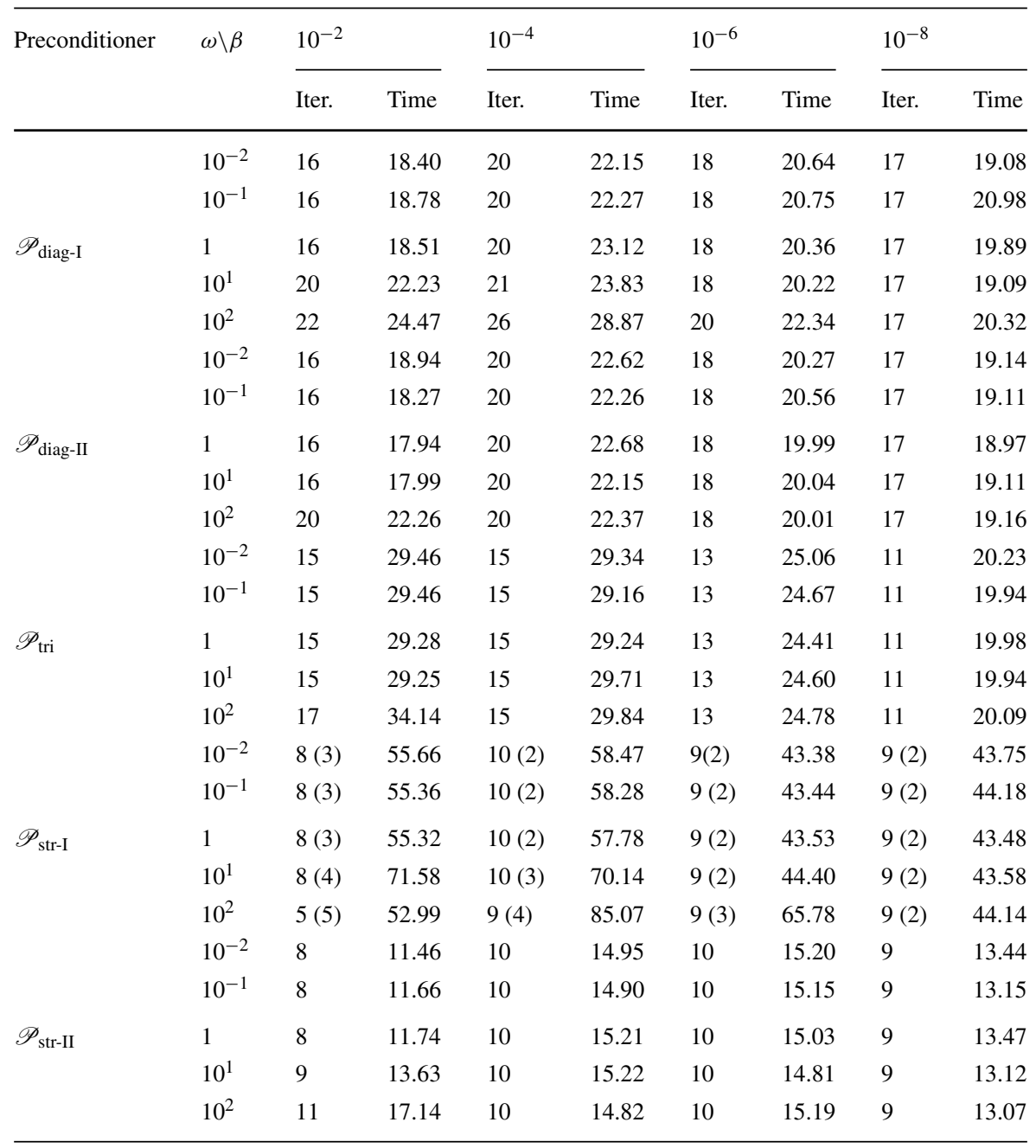

analysis in Sections 3 and 4, the $\mathscr{P}_{\text {str-II-preconditioned GMRES method performs }}$ best among all the tested methods, in terms of time. Compared with the $\mathscr{P}_{\text {diag-I }}$ and $\mathscr{P}_{\text {diag-II }}$ preconditioned MINRES methods and the $\mathscr{P}_{\text {tri }}$ preconditioned GMRES method, the $\mathscr{P}_{\text {str-II }}$ preconditioned GMRES method appears to offer advantages in terms of both iteration counts and execution time. In view of outer iteration steps, the $\mathscr{P}_{\text {str-I }}$ preconditioned inner-outer GMRES method performs better; however, the

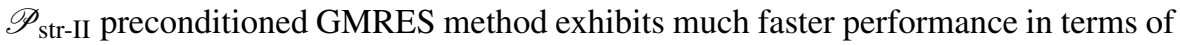
execution time.

Finally, in Fig. 1, we plot the desired state $y_{d}(x, t)$ at time $t=T$ for the problem on $64 \times 64$ grids with $\omega=1$. For comparisons, we plot the computed state $y(x, t)$ and control $u(x, t)$ obtained by the $\mathscr{P}_{\text {str-II }}$ preconditioned GMRES method for the same problem with $\beta=10^{-2}, \beta=10^{-4}, \beta=10^{-6}$, and $\beta=10^{-8}$ in Figs. 2, 3, 4, and 5, 


\section{Desired state $y_{d}$}

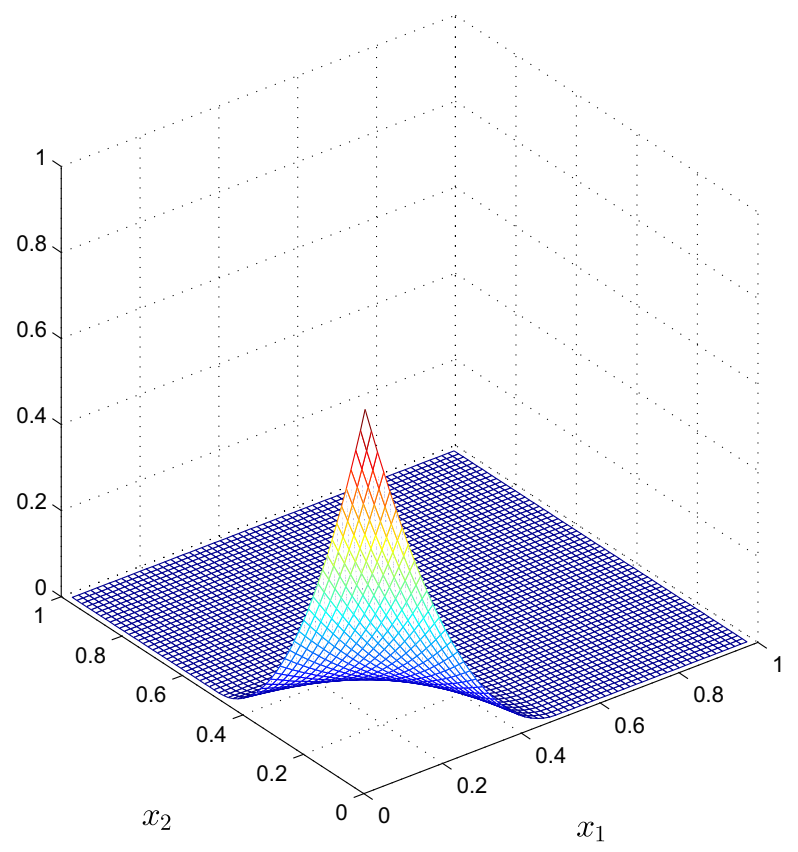

Fig. 1 Mesh plot of the desired state
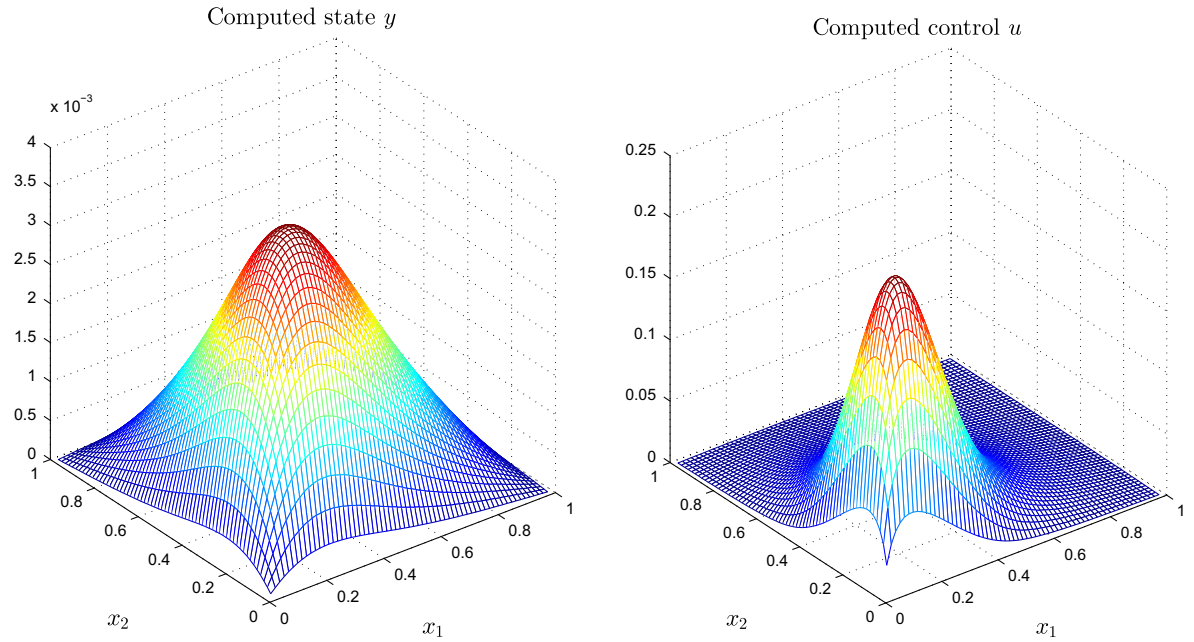

Fig. 2 Mesh plots of the computed state and control by the $\mathscr{P}_{\text {str-II }}$ preconditioned GMRES method ( $\beta=$ $10^{-2}$ ) 


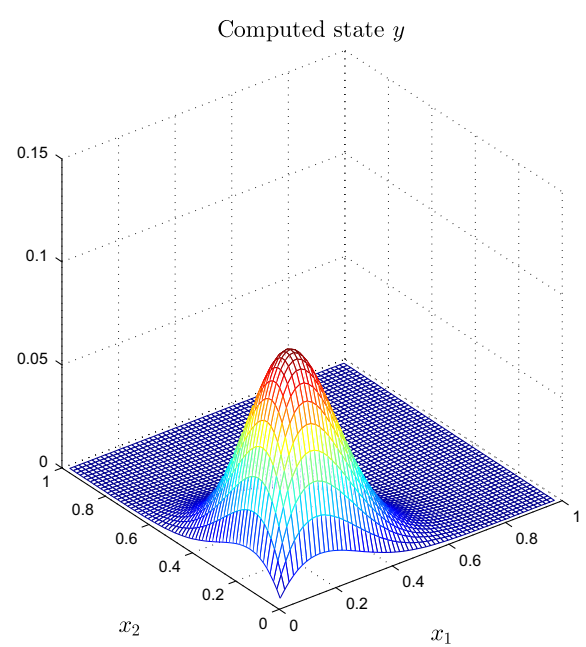

Fig. 3 Mesh plots of the computed state and control by the $\mathscr{P}_{\text {str-II }}$ preconditioned GMRES method $(\beta=$ $\left.10^{-4}\right)$

respectively. Note that we only plot the real parts of $y_{d}(x, t), y(x, t)$ and $u(x, t)$ in these figures. It shows that the computed state is quite different from the desired state when $\beta$ is not sufficiently small, i.e., $\beta=10^{-2}$ and $\beta=10^{-4}$. It becomes very close to the desired state when $\beta$ is small enough, i.e., $\beta=10^{-8}$. However, the computed norm of the control becomes larger for smaller $\beta$, but multiplied by $\beta$ the control still decreases.
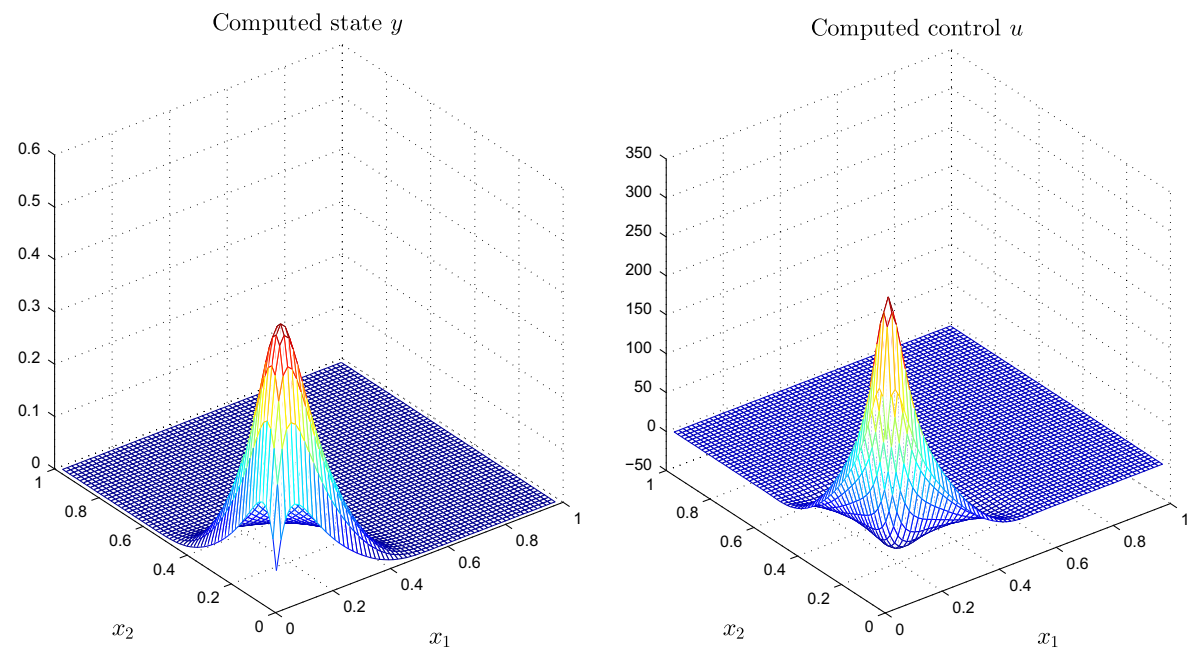

Fig. 4 Mesh plots of the computed state and control by the $\mathscr{P}_{\text {str-II }}$ preconditioned GMRES method $(\beta=$ $\left.10^{-6}\right)$ 

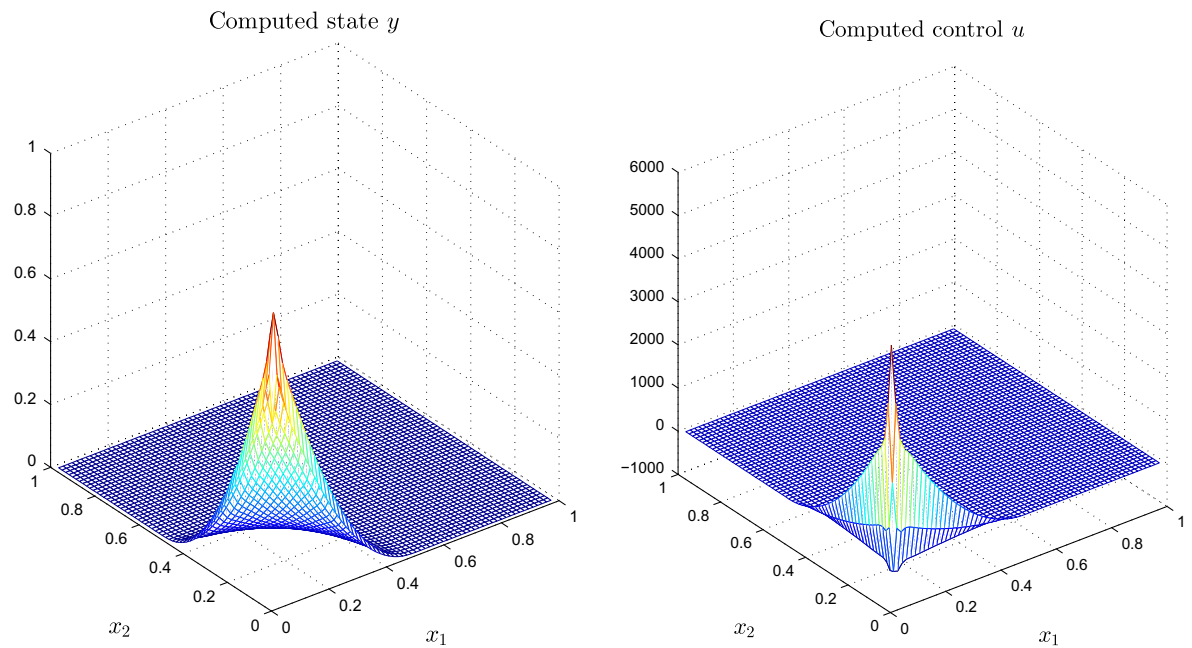

Fig. 5 Mesh plots of the computed state and control by the $\mathscr{P}_{\text {str-II }}$ preconditioned GMRES method $(\beta=$ $\left.10^{-8}\right)$

\section{Conclusions}

In this work, we construct and analyze a structured preconditioner for solving the discrete linear systems arising from the time-harmonic parabolic optimal control problem. It is proven that the eigenvalues of the corresponding preconditioned matrix are located in the interval $\left[\frac{1}{2}, 1\right]$ and, depending on the parameters, can take value even closer to unity. Numerical experiments show that the new preconditioner is robust with respect to all the involved problem parameters and performs much faster than some other tested preconditioners.

The optimal control problem we considered in this paper involves heat equation. A natural extension of our work is to apply the new structured preconditioner to tackle some more difficult problems, such as the time-harmonic eddy current control problem in [7]. In addition, as discussed in [23], the proposed preconditioner can also tackle more general algebraic problems with semidefinite or nonsymmetric matrix blocks.

However, it should be noted that the considered problem in this paper is with constant problem coefficients. In practice, more general problems with jumping coefficient arise in a wide range of scientific applications. We aim to generalize our preconditioner to solve these more difficult problems in future research.

Acknowledgements The authors are indebted to the reviewers for their careful reading and constructive advice on improving the structure of this article.

Funding information The work of Zhao-Zheng Liang is funded by the China Scholarship Council (File No. 201606180086) and by the National Natural Science Foundation of China (Grant No. 11771193). His work is performed during his visit at Uppsala University, Sweden. The work of Owe Axelsson was supported by the National Programme of Sustainability (NPU II) project IT4Innovations excellence in science-LQ1602 of the Ministry of Education, Youth and Sports of the Czech Republic. 
Open Access This article is distributed under the terms of the Creative Commons Attribution 4.0 International License (http://creativecommons.org/licenses/by/4.0/), which permits unrestricted use, distribution, and reproduction in any medium, provided you give appropriate credit to the original author(s) and the source, provide a link to the Creative Commons license, and indicate if changes were made.

\section{References}

1. Axelsson, O.: Unified analysis of preconditioning methods for saddle point matrices. Numer. Linear Algebra Appl. 22(2), 233-253 (2015)

2. Axelsson, O., Blaheta, R.: Preconditioning of matrices partitioned in $2 \times 2$ block form: eigenvalue estimates and Schwarz DD for mixed FEM. Numer. Linear Algebra Appl. 17(5), 787-810 (2010)

3. Axelsson, O., Farouq, S., Neytcheva, M.: Comparison of preconditioned Krylov subspace iteration methods for PDE-constrained optimization problems: Poisson and convection-diffusion control. Numer. Algorithms 73(3), 631-663 (2016)

4. Axelsson, O., Farouq, S., Neytcheva, M.: Comparison of preconditioned Krylov subspace iteration methods for PDE-constrained optimization problems: Stokes control. Numer. Algorithms 74(1), 1937 (2017)

5. Axelsson, O., Farouq, S., Neytcheva, M.: A preconditioner for optimal control problems, constrained by Stokes equation with a time-harmonic control. J. Comput. Appl. Math. 310, 5-18 (2017)

6. Axelsson, O., Kucherov, A.: Real valued iterative methods for solving complex symmetric linear systems. Numer. Linear Algebra Appl. 7(4), 197-218 (2000)

7. Axelsson, O., Lukáš, D.: Preconditioning methods for eddy-current optimally controlled timeharmonic electromagnetic problems. Submitted to J. Numer. Math. (2017)

8. Axelsson, O., Neytcheva, M.: A general approach to analyse preconditioners for two-by-two block matrices. Numer. Linear Algebra Appl. 20(5), 723-742 (2013)

9. Axelsson, O., Neytcheva, M., Ahmad, B.: A comparison of iterative methods to solve complex valued linear algebraic systems. Numer. Algorithms 66(4), 811-841 (2014)

10. Benzi, M., Bertaccini, D.: Block preconditioning of real-valued iterative algorithms for complex linear systems. IMA J. Numer. Anal. 28(3), 598-618 (2008)

11. Benzi, M., Golub, G.H., Liesen, J.: Numerical solution of saddle point problems. Acta Numer. 14, 1-137 (2005)

12. Collis, S.S., Heinkenschloss, M.: Analysis of the streamline upwind/Petrov Galerkin method applied to the solution of optimal control problems CAAM TR02-01 (2002)

13. Day, D., Heroux, M.A.: Solving complex-valued linear systems via equivalent real formulations. SIAM J. Sci. Comput. 23(2), 480-498 (2001)

14. De los Reyes, J.C.: Numerical PDE-constrained optimization. Springer, Berlin (2015)

15. Elman, H.C., Ramage, A., Silvester, D.J.: Algorithm 866: IFISS, a Matlab toolbox for modelling incompressible flow. ACM Trans. Math. Softw. 33(2), 14 (2007)

16. Elman, H.C., Ramage, A., Silvester, D.J.: IFISS: a computational laboratory for investigating incompressible flow problems. SIAM Rev. 56(2), 261-273 (2014)

17. Greenbaum, A.: Iterative methods for solving linear systems, SIAM (1997)

18. Hinze, M., Pinnau, R., Ulbrich, M., Ulbrich, S.: Optimization with PDE constraints, mathematical modelling: theory and applications, vol. 23. Springer, New York (2008)

19. Kollmann, M., Kolmbauer, M., Langer, U., Wolfmayr, M., Zulehner, W.: A robust finite element solver for a multiharmonic parabolic optimal control problem. Comput. Math. Appl. 65(3), 469-486 (2013)

20. Kolmbauer, M., Langer, U.: A robust preconditioned MinRes solver for distributed time-periodic eddy current optimal control problems. SIAM J. Sci. Comput. 34(6), B785-B809 (2012)

21. Krendl, W., Simoncini, V., Zulehner, W.: Stability estimates and structural spectral properties of saddle point problems. Numer. Math. 124(1), 183-213 (2013)

22. Langer, U., Wolfmayr, M.: Multiharmonic finite element analysis of a time-periodic parabolic optimal control problem. J. Numer. Math. 21(4), 265-300 (2013)

23. Liang, Z.Z., Axelsson, O., Neytcheva, M.: A robust structured preconditioner for time-harmonic parabolic optimal control problems. Technical report Uppsala University, Department of Information Technology, http://www.it.uu.se/research/publications/reports/, 2017-014/08 (2017)

24. Mardal, K.A., Winther, R.: Preconditioning discretizations of systems of partial differential equations. Numer. Linear Algebra Appl. 18(1), 1-40 (2011) 
25. Notay, Y.: A new analysis of block preconditioners for saddle point problems. SIAM J. Matrix Anal. Appl. 35(1), 143-173 (2014)

26. Paige, C.C., Saunders, M.A.: Solution of sparse indefinite systems of linear equations. SIAM J. Numer. Anal. 12(4), 617-629 (1975)

27. Pearson, J.W., Wathen, A.J.: A new approximation of the schur complement in preconditioners for PDE-constrained optimization. Numer. Linear Algebra Appl. 19(5), 816-829 (2012)

28. Rees, T., Dollar, H.S., Wathen, A.J.: Optimal solvers for PDE-constrained optimization. SIAM J. Sci Comput. 32(1), 271-298 (2010)

29. Saad, Y.: Iterative methods for sparse linear systems. SIAM (2003)

30. Saad, Y., Schultz, M.H.: GMRES: A generalized minimal residual algorithm for solving nonsymmetric linear systems. SIAM J. Sci. Stat. Comput. 7(3), 856-869 (1986)

31. Tröltzsch, F.: Optimal control of partial differential equations: theory, methods and applications, vol. 112. American Mathematical Soc., Providence (2010)

32. Vassilevski, P.: Multilevel block factorization preconditioners: matrix-based analysis and algorithms for solving finite element equations. Springer (2008)

33. Zheng, Z., Zhang, G.-F., Zhu, M.-Z.: A block alternating splitting iteration method for a class of block two-by-two complex linear systems. J. Comput. Appl. Math. 288, 203-214 (2015)

34. Zheng, Z., Zhang, G.-F., Zhu, M.-Z.: A note on preconditioners for complex linear systems arising from PDE-constrained optimization problems. Appl. Math. Lett. 61, 114-121 (2016)

35. Zulehner, W.: Nonstandard norms robust estimates for saddle point problems. SIAM J Nonstandard Matrix Anal. Appl. 32(2), 536-560 (2011) 